\title{
Determinação ultra-sonográfica do sexo fetal pela medida dos ângulos do apêndice genital
}

Sonographic determination of fetal gender by measurement of the angles of the genital tubercle

Victor Bunduki ${ }^{1}$, Maria de Lourdes Brizot² ${ }^{2}$ Javier Miguelez ${ }^{3}$, Cleisson Fábio Andrioli Peralta ${ }^{4}$, Alberto Jorge Monteiro de La Veja ${ }^{5}$, Marcelo Zugaib ${ }^{6}$

\section{RESUMO}

Objetivo: avaliar a acurácia da ultra-sonografia para predição do sexo fetal entre a $11^{\text {a }}$ e a $13^{\text {a }}$ semana e 6 dias por meio da medida dos ângulos anterior e posterior do apêndice genital. Métodos: os ângulos anterior e posterior do apêndice genital foram medidos em corte sagital de 455 fetos entre a $11^{\mathrm{a}}$ e a $13^{\mathrm{a}}$ semana e 6 dias. A probabilidade de predição correta do sexo fetal (confirmado após o nascimento) foi categorizada de acordo com a medida dos ângulos, idade gestacional e comprimento crânio-caudal. Os pontos de corte de melhor acurácia para predizer o sexo fetal foram obtidos por meio de curva ROC. A variação interobservador foi avaliada pelo método de Bland-Altman. Resultados: a taxa de predição correta do sexo fetal se elevou com a idade gestacional e comprimento crânio-nádegas. Utilizando o ponto de corte de 42 graus para o ângulo anterior (curva ROC), a predição correta do sexo fetal ocorreu em $72 \%$ dos fetos da $11^{\mathrm{a}}$ à $11^{\mathrm{a}}$ semana e 6 dias, $86 \%$ da $12^{\mathrm{a}}$ à $12^{\mathrm{a}}$ semana e 6 dias e $88 \%$ da $13^{\mathrm{a}}$ à $13^{\mathrm{a}}$ semana e 6 dias. As taxas de predição obtidas com o ângulo posterior (ponto de corte de 24 graus) foram, respectivamente: 70,87 e $87 \%$. A avaliação da variação interobservador revelou diferença média entre medidas pareadas de 15,7 e 9 graus para as medidas dos ângulos posterior e anterior, respectivamente. Conclusão: a acurácia da medida dos ângulos do apêndice genital na predição do sexo fetal foi elevada a partir da $12^{\mathrm{a}}$ semana de gestação mas ainda insuficiente para sua aplicação em gestações com risco de doenças graves ligadas ao cromossomo X.

PALAVRAS-CHAVE: Determinação do sexo (análise); Primeiro trimestre da gravidez; Gravidez normal; Diagnóstico prénatal; Ultrasonografia pré-natal

\section{ABSTRACT}

Purpose: to evaluate the accuracy of fetal gender prediction at 11 to 13 weeks and 6 days by measuring the anterior and posterior genital tubercle angles. Methods: the anterior and posterior genital tubercle angles were measured in a midsagittal plane in 455 fetuses from 11 to 13 weeks and 6 days. The probability of a correct fetal sex prediction (confirmed after birth) was categorized in accordance with the angle measurements, gestational age and crump-rump length. The optimal accuracy cutoffs were derived from a ROC-plot. The interobserver variability was evaluated by a Bland-Altman plot. Results: the correct fetal sex prediction rate increased with gestational age and crump-rump length. Using a 42-degree anterior angle as a cutoff, a correct fetal sex prediction occurred in $72 \%$ of the fetuses from 11 to 11 weeks and 6 days, $86 \%$ from 12 to 12 weeks and 6 days and $88 \%$ from 13 to 13 weeks and 6 days. Using a 24-degree posterior angle as a cutoff, a correct fetal gender prediction occurred in 70, 87 and $87 \%$, respectively. The interobserver variability evaluation revealed a mean difference between paired measurements of 15.7 and 9 degrees for the posterior and anterior angles, respectively. Conclusion: the measurement of the genital tubercle angles showed a high accuracy in correctly predicting the fetal sex from the 12th week of gestation on. However, accuracy was still not high enough for clinical use in pregnancies at risk of serious X-linked diseases.

KEYWORDS: Sex determination (analysis); Pregnancy trimester, first; Pregnancy; Prenatal diagnosis; Ultrasonography, prenatal

1 Professor Livre Docente e Associado da FMUSP, responsável pelo setor de Medicina Fetal da Clinica Obstétrica do Hospital das Clínicas da Faculdade de Medicina da Universidade de São Paulo - USP - São Paulo (SP) - Brasil.

2 Assistente da Clinica Obstétrica do HCFMUSP, responsável pelo setor de Ultra-sonografia Clinica Obstétrica do Hospital das Clinicas da Faculdade de Medicina da Universidade de São Paulo - USP - São Paulo (SP) - Brasil.

3 Pós-graduando da Faculdade de Medicina da Universidade de São Paulo - USP - São Paulo (SP) - Brasil.

4 Mestre e Doutor em Medicina pela Faculdade de Medicina da Universidade de São Paulo - USP - São Paulo (SP) - Brasil.

5 Aluno do $5^{\circ}$ ano da Faculdade de Medicina da Universidade de São Paulo - USP - São Paulo (SP) - Brasil.

6 Professor Titular da Disciplina de Obstetrícia do Departamento de Obstetricia e Ginecologia da Faculdade de Medicina da Universidade de São Paulo Urofessor Titular da Disciplina de

Setor de Medicina Fetal do Departamento de Obstetrícia e Ginecologia do Hospital das Clínicas da Faculdade de Medicina da Universidade de São Paulo. Correspondência: Victor Bunduki

Rua Oswaldo Moreira Pompeo, n 61 - Paraíso - 04001-115 - São Paulo - SP - e-mail: victor.bunduki@hcnet.usp.br

Recebido em: 18/10/2005

Aceito com modificações em: 17/6/2005

RevBras Ginecol Obstet. 2005;27(6):310-5 


\section{Introdução}

A avaliação da genitália externa é parte rotineira no estudo morfológico fetal no segundo trimestre da gravidez ${ }^{1}$. Mais recentemente, a evolução da tecnologia ultra-sonográfica tem permitido o diagnóstico cada vez mais precoce do sexo fetal, até mesmo no final do primeiro trimestre da gestação ${ }^{2-4}$.

A determinação do sexo fetal no primeiro trimestre é de fundamental importância em gestantes com risco genético de hiperplasia congênita de supra-renal ${ }^{2-5}$ (de herança autossômica recessiva) e nas anomalias genéticas ligadas ao cromossomo X, como a distrofia muscular de Duchenne e a hemofilia. A confirmação do sexo masculino na primeira situação descrita e do sexo feminino nas duas últimas exige tradicionalmente a realização da biópsia de vilo corial, a qual não é isenta de risco de perda gestacional ${ }^{4}$.

O diagnóstico ultra-sonográfico do sexo fetal no primeiro trimestre é baseado na direção que os precursores do pênis e clitóris (denominados apêndice genital) apontam, em corte sagital: cranial para os fetos de sexo masculino e caudal para o sexo feminino. À medida que se eleva a idade gestacional (IG), o apêndice genital assume direção progressivamente mais cranial nos fetos de sexo masculino, mantendo-se inalterada nos fetos de sexo feminino. Por este motivo, a diferenciação entre o sexo fetal masculino e feminino à ultra-sonografia é mais fidedigna quanto maior a $\mathrm{IG}^{3}$.

É consenso que a ultra-sonografia tem acurácia muito baixa (cerca de $60 \%$ ) no diagnóstico do sexo fetal antes da $12^{a}$ semana de gestação ${ }^{2-8}$. Entre a $12^{\mathrm{a}}$ e a $13^{\mathrm{a}}$ semana, a literatura apresenta resultados variados (75 a 99\%), com boa acurácia (cerca de $100 \%$ ) apenas a partir da $14^{\mathrm{a}}$ semana de gestação $^{2-8}$. Sob a ótica da embriologia, esse período pode ser considerado tardio, já que a diferenciação da genitália fetal se inicia pelo menos duas semanas antes ${ }^{9}$. Também é tardio sob o ponto de vista clínico, uma vez que a biópsia de vilo corial permite um diagnóstico definitivo até três semanas antes ${ }^{3}$.

Até o presente, os estudos envolvendo a predição do sexo fetal à ultra-sonografia no primeiro trimestre da gestação se limitaram a avaliar a direção do apêndice genital de forma subjetiva (cranial ou caudal $)^{2-8}$. A existência de casos nos quais o apêndice genital assume direção intermediária poderia explicar parte dos erros no diagnóstico do sexo fetal antes da $14^{\mathrm{a}}$ semana de gestação.

Este estudo pretende introduzir critérios objetivos para avaliar a direção do apêndice genital. É proposta a mensuração dos ângulos que o apêndice genital forma com dois pontos de referência fetais (denominados ângulos anterior e posterior do apêndice genital) em fetos de ambos os sexos. Será ainda avaliada a sua reprodutibilidade (variação interobservador), o que é essencial para sua validação como método diagnóstico. Pretende-se avaliar se a utilização das medidas dos ângulos anterior e posterior do apêndice genital, ao minimizar a subjetividade na avaliação do apêndice genital, permitiu melhorar a acurácia da predição ultra-sonográfica do sexo fetal entre a $11^{\mathrm{a}}$ e a $13^{\mathrm{a}}$ semana e 6 dias de gestação.

Métodos

De abril de 2000 a junho de 2003, foram incluídas no estudo 508 gestantes atendidas no Setor de Medicina Fetal da Clínica Obstétrica do Hospital das Clinicas da Faculdade de Medicina da Universidade de São Paulo e na clínica particular do primeiro autor. O estudo foi desenvolvido como coorte prospectivo. As gestações estavam entre a $11^{\mathrm{a}}$ e a $13^{\mathrm{a}}$ semana e 6 dias, tendo sido agendadas para avaliação quanto ao risco de trissomias utilizando-se o programa de medida da translucência nucal.

Os critérios de inclusão foram: fetos de gestação única com IG entre a $11^{\mathrm{a}}$ e a $13^{\mathrm{a}}$ semana e 6 dias de gestação e comprimento crânio-nádegas (CCN) entre 45 e $84 \mathrm{~mm}$. Os critérios de exclusão foram: impossibilidade técnica de obter imagem nítida do apêndice genital e perda de seguimento da gestação (impossibilidade de confirmar o sexo fetal após o nascimento). De um total de 508 casos, 455 casos foram avaliados de maneira completa e 53 foram excluídos. Destes, em 23 (4,5\%) houve impossibilidade técnica de se obter imagem nítida do apêndice genital, devido à obesidade materna ou interposição do cordão umbilical. Ainda, em 30 casos $(5,9 \%)$ não foi possivel confirmar o sexo após o nascimento (perda de seguimento).

Todos os exames foram realizados por via transabdominal, utilizando-se aparelhos de dois a oito $\mathrm{MHz}$ (Toshiba Corevision, Toshiba 270, GE Logic 700). Uma fotografia foi obtida em todos os casos, documentando o feto em corte sagital estrito, ocupando pelo menos $75 \%$ da tela. As fotografias foram analisadas em um segundo momento por um único operador, sem conhecimento do sexo fetal.

Utilizando caneta esferográfica foram traçadas três linhas: a primeira entre a base do apêndice genital e a inserção fetal do cordão umbilical; a segunda sobre o eixo do próprio apêndice genital (entre a sua base e seu ápice) e a terceira, paralela à coluna lombo-sacral fetal. Posteriormente, procedeu-se à medição dos ângulos anterior e posterior do apêndice genital, utilizando régua e transferidor sobre a fotografia. Mediu-se em graus o ângulo formado entre o eixo do apêndice genital e a reta que passa pela inserção abdominal do cordão e a base do apêndice, na porção caudal e anterior, chamado de ângulo anterior. O ângulo posterior foi formado pelo eixo do apêndice genital e uma reta que passa paralela à coluna lombo-sacral fetal, na porção caudal e anterior (Figura 1).

Rev Bras Ginecol Obstet. 2005; 27(6):310-5 


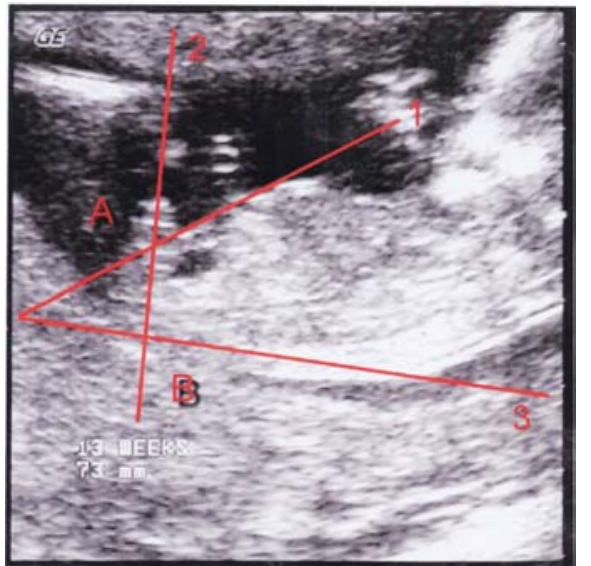

Figura 1 - Foto em corte sagital de um feto do sexo masculino na $13^{\mathrm{a}}$ semana de gestação (comprimento crânio-nádegas de $73 \mathrm{~mm}$ ). Notam-se três retas: (1) da base do apêndice genital à inserção abdominal do cordão umbilical; (2) eixo do apêndice genital; (3) eixo paralelo à coluna lombo-sacra. $O$ ângulo $A$ (entre as retas 1 e 2 ) mede 135 graus e 0 ângulo $B$ (entre as retas 2 e 3 ) mede 90 graus.

Após completada a mensuração dos ângulos, cada caso foi incluído, juntamente com a IG e CCN, em planilha eletrônica, na qual foi relacionado ao respectivo sexo após o nascimento, confirmado por meio de contato telefônico com os pais ou exame do recém-nascido e seu registro de berçário. O desempenho diagnóstico dos ângulos anterior e posterior do apêndice genital foi avaliado por meio de curva do tipo receiver-operator characteristic (ROC). Foram calculados os pontos de corte para estes ângulos que apresentaram melhor balanço entre sensibilidade e taxa de falso-positivos.

Em seguida, foram calculadas, utilizando-se os pontos de corte dos ângulos do apêndice genital obtidos por meio da curva ROC, as taxas de predição correta do sexo fetal, categorizadas por sexo e IG/ CCN: de 11 a 11 semanas e 6 dias (CCN: 45,0-55,3 $\mathrm{mm}$ ), de 12 a 12 semanas e 6 dias (CCN: 55,4-67,9 $\mathrm{mm}$ ) e de 13 a 13 semanas e 6 dias (CCN: 68,0-84,0 $\mathrm{mm})$. Foi também calculada a probabilidade de predizer corretamente o sexo fetal masculino, de acordo com diferentes intervalos de valores dos ângulos anterior e posterior, e de acordo com a IG e CCN.

Os últimos trinta casos da série, avaliados em dias consecutivos, foram escolhidos para avaliar a variação interobservador. Nestes casos, após um primeiro operador realizar o exame da paciente e ter obtido fotografia do feto em corte sagital, conforme descrito anteriormente, um segundo operador (sem acesso ao resultado ou registro fotográfico do primeiro exame) foi convidado a obter uma segunda fotografia do feto em corte sagital, obedecendo aos mesmos critérios. As fotografias foram guardadas em envelopes fechados e separados e os ângulos anterior e posterior do apêndice genital foram avaliados de forma independente. Cada operador mediu os ângulos da fotografia correspondente a seu próprio exame, da mesma forma descrita anteriormente, não tendo acesso aos resultados do outro examinador. Num se- gundo momento, as medidas pareadas dos dois ângulos foram digitadas em planilha eletrônica, com cálculo da diferença média das medidas pareadas e seus respectivos limites de tolerância de 95\%, segundo o método de Bland-Altman ${ }^{10}$.

O estudo foi aprovado pelo Comitê de Ética em Pesquisa do Hospital das Clinicas da Faculdade de Medicina da Universidade de São Paulo e todos os exames foram realizados após assinatura do termo de consentimento informado.

\section{Resultados}

Os niveis de corte para os ângulos do apêndice genital que apresentaram melhor balanço entre sensibilidade e taxa de falso-positivos, obtidos por meio da curva ROC, foram: 42 graus para o ângulo anterior e 24 graus para o ângulo posterior (Figuras 2 e 3).

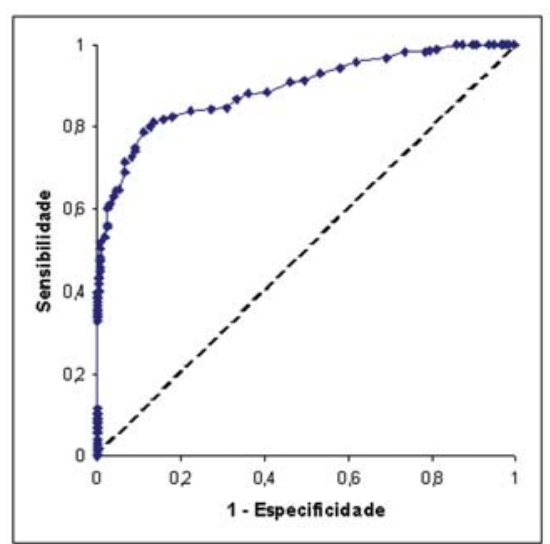

Figura 2 - Curva ROC ilustrando a relação entre sensibilidade e o inverso da especificidade de diferentes níveis de corte (de dez a 140 graus) do ângulo anterior do apêndice genital na detecção de 216 fetos de sexo masculino em um total de 455. No caso, a sensibilidade (eixo $Y$ ) expressa a proporcão de fetos do sexo masculino, corretamente identificados como masculinos. Já o inverso da especificidade, também conhecida como taxa de falso-positivos (eixo X), expressa a proporção de fetos conhecida como taxa de falso-positivos (eixo X), expressa a proporção de fetos relação entre sensibilidade e especificidade foi 0 ângulo de 42 graus.

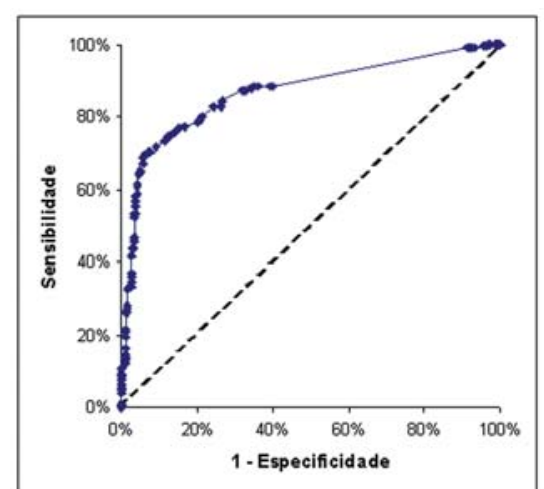

Figura 3 - Curva ROC ilustrando a relação entre sensibilidade e o inverso da especificidade de diferentes níveis de corte (de -40 a 100 graus) do ângulo posterior do apêndice genital na detecção de 216 fetos de sexo masculino em um total de 455 . No caso, a sensibilidade (eixo Y) expressa a proporção de fetos do sexo masculino, corretamente identificados como masculinos. Já o inverso da especificidade, também conhecida como taxa de falso-positivos (eixo X), expressa a proporção de fetos erroneamente identificados como masculinos. 0 ponto de corte que apresentou melhor relação entre sensibilidade e especificidade foi 0 ângulo de 24 graus. 
Assim, um ângulo anterior superior ou igual a 42 graus prediz corretamente o sexo masculino em $62 \%$ dos casos entre 11 e 11 semanas e 6 dias, $86 \%$ entre 12 e 12 semanas e 6 dias e $91 \%$ entre 13 e 13 semanas e 6 dias. Por outro lado, um ângulo anterior inferior a 42 graus diagnostica corretamente o sexo feminino em 81,86 e $84 \%$ dos casos entre 11 e 11 semanas e 6 dias, 12 e 12 semanas e 6 dias e 13 e 13 semanas e 6 dias, respectivamente (Tabela 1 ).

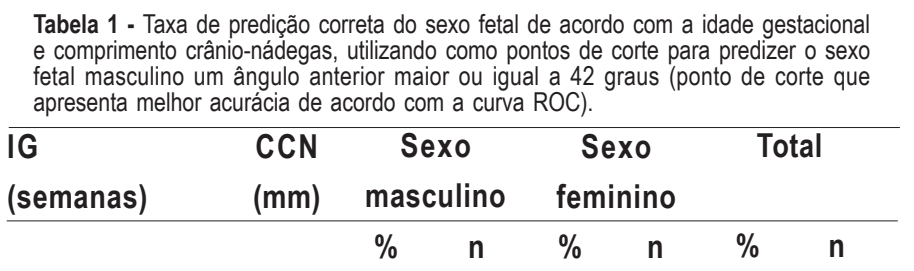

\begin{tabular}{|c|c|c|c|}
\hline $11^{\mathrm{a}}$ à $11^{\mathrm{a}}$ e 6 dias & 45,0 a $55,362 \quad(36 / 58)$ & $81(56 / 69)$ & $72(92 / 127)$ \\
\hline $12^{\mathrm{a}}$ à $12^{\mathrm{a}}$ e 6 dias & 55,4 a $67,986 \quad(66 / 76)$ & $86(80 / 93)$ & $86(146 / 169)$ \\
\hline $13^{\mathrm{a}}$ à $13^{\mathrm{a}}$ e 6 dias & 68,0 a $84,091 \quad(75 / 82)$ & $84 \quad(65 / 77)$ & $88(140 / 159)$ \\
\hline Total & $82(177 / 216)$ & $84(201 / 239)$ & $83(378 / 455)$ \\
\hline
\end{tabular}

Os valores limites do ângulo anterior acima do qual afirmou-se o sexo masculino e abaixo do qual afirmou-se o sexo feminino foram de 70 graus e 20 graus, respectivamente. Dessa maneira, nenhum feto de sexo masculino apresentou ângulo anterior inferior a 20 graus, ou seja, um ângulo anterior abai-
Já um ângulo posterior superior ou igual a 24 graus prediz corretamente o sexo masculino em $43 \%$ dos casos entre 11 e 11 semanas e 6 dias, $76 \%$ de acerto entre 12 e 12 semanas e 6 dias e $84 \%$ entre 13 e 13 semanas e 6 dias. Um ângulo posterior inferior a 24 graus indica corretamente o sexo feminino em 93, 95 e $90 \%$ dos casos entre 11 e 11 semanas e 6 dias, 12 e 12 semanas e 6 dias e 13 e 13 semanas e 6 dias, respectivamente (Tabela 2).

Tabela 2 - Taxa de predição correta do sexo fetal de acordo com a idade gestacional e comprimento crânio-nádegas, utilizando como pontos de corte para predizer o sexo fetal masculino um ângulo posterior superior ou igual a 24 graus (ponto de corte que apresenta melhor acurácia de acordo com a curva ROC).

\begin{tabular}{lcccc}
\hline IG & CCN & Sexo & Sexo & Total \\
& (semanas) & masculino & feminino & \\
& $(\mathrm{mm})$ & $\%$ & $\mathrm{n}$ & $\%$
\end{tabular}

\begin{tabular}{lccccccc} 
& $(\mathbf{m m})$ & $\%$ & $\mathbf{n}$ & $\%$ & $\mathbf{n}$ & $\%$ & $\mathbf{n}$ \\
\hline $11^{\mathrm{a}}$ à $11^{\mathrm{a}}$ e 6 dias & 45,0 a 55,3 & 43 & $(25 / 58)$ & 93 & $(64 / 69)$ & 70 & $(89 / 127)$ \\
$12^{\mathrm{a}}$ à $12^{\mathrm{a}}$ e 6 dias & 55,4 a 67,9 & 76 & $(58 / 76)$ & 95 & $(89 / 93)$ & 87 & $(147 / 169)$ \\
$13^{\mathrm{a}}$ à $13^{\mathrm{a}}$ e 6 dias & 68,0 a 84,0 & 84 & $(69 / 82)$ & 90 & $(69 / 77)$ & 87 & $(138 / 159)$ \\
Total & & 70 & $(152 / 216)$ & 93 & $(222 / 239)$ & 71 & $(323 / 455)$
\end{tabular}

\section{IG: Idade gestacional.}

CCN: Comprimento crânio-nádegas.

$\mathrm{n}$ : número de casos.

Tabela 3 - Probabilidade de sexo fetal masculino de acordo com o ângulo anterior encontrado e segundo a idade gestacional e comprimento crânio-nádegas.

\begin{tabular}{|c|c|c|c|c|c|c|c|c|}
\hline \multirow{2}{*}{$\begin{array}{l}\text { Ângulo } \\
\text { graus }\end{array}$} & \multicolumn{2}{|c|}{$\begin{array}{l}\text { IG: } 11^{\mathrm{a}} \text { à } 11^{\mathrm{a}} \mathrm{s} \text { e } 6 \mathrm{~d} \\
(\mathrm{CCN}: 45,0-55,3 \mathrm{~mm})\end{array}$} & \multicolumn{2}{|c|}{$\begin{array}{l}\text { IG: } 11^{\mathrm{a}} \text { à } 11^{\mathrm{a}} \text { s e } 6 \mathrm{~d} \\
\text { (CCN: } 55,4-67,9 \mathrm{~mm})\end{array}$} & \multicolumn{2}{|c|}{$\begin{array}{l}\text { IG: } 11^{\mathrm{a}} \text { à } 11^{\mathrm{a}} \text { s e } 6 \mathrm{~d} \\
\text { (CCN: } 68,0-84,0 \mathrm{~mm})\end{array}$} & \multicolumn{2}{|c|}{ Total } \\
\hline & $\%$ & $\mathrm{n}$ & $\%$ & $\mathrm{n}$ & $\%$ & $\mathrm{n}$ & $\%$ & $\mathrm{n}$ \\
\hline $0-20$ & 0 & $(0 / 4)$ & 0 & $(0 / 11)$ & 0 & $(0 / 7)$ & 0 & $(0 / 22)$ \\
\hline $21-30$ & 24 & $(7 / 29)$ & 7 & $(2 / 29)$ & 9 & $(3 / 32)$ & 13 & $(12 / 90)$ \\
\hline $31-40$ & 35 & $(15 / 43)$ & 15 & $(7 / 47)$ & 13 & $(4 / 32)$ & 21 & $(26 / 122)$ \\
\hline $41-50$ & 57 & $(13 / 23)$ & 46 & $(12 / 26)$ & 60 & $(12 / 20)$ & 53 & $(37 / 69)$ \\
\hline $51-60$ & 64 & $(9 / 14)$ & 94 & $(15 / 16)$ & 75 & $(12 / 16)$ & 78 & $(36 / 46)$ \\
\hline $61-70$ & 100 & $(4 / 4)$ & 100 & $(8 / 8)$ & 78 & $(7 / 9)$ & 90 & $(19 / 21)$ \\
\hline$>70$ & 100 & $(10 / 10)$ & 100 & $(32 / 32)$ & 100 & $(43 / 43)$ & 100 & $(85 / 85)$ \\
\hline
\end{tabular}

IG: Idade gestacional.

CCN: Comprimento crânio-nádegas.

n: número de casos.

s: semana de gestação.

d: dias.

O valor limite do ângulo posterior acima do qual afirmou-se ser o sexo masculino foi de 60 graus e o ângulo posterior abaixo do qual se afirmou ser o sexo feminino foi de zero grau. Dessa maneira, nenhum feto de sexo masculino apresentou ângulo posterior de zero grau, ou seja, um ângulo poste- xo de 20 graus indicou sexo fetal feminino. Da mesma forma, nenhum feto de sexo feminino apresentou ângulo anterior superior a 70 graus, o que permite concluir que um ângulo anterior superior a 70 graus é praticamente equivalente ao diagnóstico de sexo fetal masculino (Tabela 3 ). 
Tabela 4 - Probabilidade de sexo fetal masculino de acordo com 0 ângulo posterior encontrado e segundo a idade gestacional e comprimento crânio-nádegas.

\begin{tabular}{|c|c|c|c|c|c|c|c|c|}
\hline \multirow{2}{*}{$\begin{array}{l}\text { Ângulo } \\
\text { graus }\end{array}$} & \multicolumn{2}{|c|}{$\begin{array}{l}\text { IG: } 11^{\mathrm{a}} \text { à } 11^{\mathrm{a}} \text { s e } 6 \mathrm{~d} \\
(\mathrm{CCN}: 45,0-55,3 \mathrm{~mm})\end{array}$} & \multicolumn{2}{|c|}{$\begin{array}{l}\text { IG: } 11^{\mathrm{a}} \text { à } 11^{\mathrm{a}} \text { s e } 6 \mathrm{~d} \\
(\mathrm{CCN}: 55,4-67,9 \mathrm{~mm})\end{array}$} & \multicolumn{2}{|c|}{$\begin{array}{l}\text { IG: } 11^{\mathrm{a}} \text { à } 11^{\mathrm{a}} \text { s e } 6 \mathrm{~d} \\
\text { (CCN: } 68,0-84,0 \mathrm{~mm})\end{array}$} & \multicolumn{2}{|c|}{ Total } \\
\hline & $\%$ & $n$ & $\%$ & $n$ & $\%$ & $n$ & $\%$ & $n$ \\
\hline$<0$ & 0 & $(0 / 1)$ & 0 & $(0 / 10)$ & 0 & $(0 / 10)$ & 0 & $(0 / 21)$ \\
\hline $0-20$ & 33 & $(31 / 93)$ & 17 & $(15 / 89)$ & 17 & $(11 / 66)$ & 23 & $(57 / 248)$ \\
\hline $21-30$ & 86 & $(6 / 7)$ & 61 & $(11 / 18)$ & 50 & $(9 / 18)$ & 60 & $(26 / 43)$ \\
\hline $31-40$ & 86 & $(6 / 7)$ & 100 & $(13 / 13)$ & 93 & $(13 / 14)$ & 94 & $(32 / 34)$ \\
\hline $41-50$ & 75 & $(9 / 12)$ & 96 & $(22 / 23)$ & 100 & $(9 / 9)$ & 91 & $(40 / 44)$ \\
\hline $51-60$ & 80 & $(4 / 5)$ & 86 & $(6 / 7)$ & 89 & $(16 / 18)$ & 87 & $(26 / 30)$ \\
\hline$>60$ & 100 & $(2 / 2)$ & 100 & $(9 / 9)$ & 100 & $(24 / 24)$ & 100 & $(35 / 35)$ \\
\hline
\end{tabular}

IG: Idade gestacional.

CCN: Comprimento crânio-nádegas.

n: número de casos.

s: semana de gestação.

d: dias.

Salientamos que a certeza de sexo fetal baseada nestes dados expostos acima ocorreu em 107 casos $(23,5 \%)$ para o ângulo anterior e em 56 casos $(12,3 \%)$ para o ângulo posterior em qualquer IG e CCN.

A variação interobservadores, segundo o método de Bland e Altman ${ }^{10}$, encontrou uma diferença média das medidas pareadas de 15,7 graus para o ângulo posterior (limites de tolerância de 95\% de $-1,7$ a 33 graus) e de nove graus para o anterior (limites de tolerância de $95 \%$ de $-6,5$ a 24,5 graus).

\section{Discussão}

A contribuição original deste trabalho foi a introdução de critérios objetivos para avaliar o apêndice genital, com razoável reprodutibilidade e que independem da experiência do operador em diagnosticar o sexo fetal em idade gestacional precoce. Isso pode facilitar o aprendizado e difusão da técnica, entre quaisquer operadores habilitados em ultra-sonografia.

Entretanto, não parece que a introdução das medidas dos ângulos do apêndice genital tenha contribuido para melhorar sensivelmente a acurácia do diagnóstico do sexo fetal. A avaliação ultrasonográfica do apêndice genital apresentou acurácia baixa para predição do sexo fetal $(70$ e $72 \%)$ na $11^{\text {a }}$ semana de gestação e apenas razoável na $13^{\mathrm{a}}$ semana (87 e 88\%), o que não difere muito do que já foi descrito na literatura ${ }^{2-8}$. É fato também que em decorrência de fatores técnicos (obesidade materna, posição fetal, interposição do cordão umbilical) em alguns casos (4,5\% neste trabalho) a ultrasonografia não permite adequada avaliação da genitália fetal. O uso de técnicas tridimensionais não parece trazer beneficios nestas situações ${ }^{2}$.
A despeito de suas limitações, a mensuração dos ângulos do apêndice genital tem interesse na prática clínica. Após consultar as tabelas de probabilidade descritas neste trabalho, é possivel fornecer à gestante uma probabilidade individualizada do sexo fetal, de acordo com a IG, CCN e ângulo encontrado. Por exemplo, uma gestante com IG de 12 semanas (CCN: 56 mm) e um ângulo anterior de 55 graus apresenta probabilidade de ter um feto masculino de $94 \%$. O uso de estatística e probabilidades no contexto clínico é uma forma elegante de apresentar informações aos pacientes e uma tendência crescente na medicina.

Há mesmo alguns casos nos quais o ângulo encontrado permitiu afirmar com certeza o sexo fetal (por exemplo, ângulo posterior superior a 60 graus). Entretanto, isso ocorreu em apenas um pequeno número de casos (cerca de $23 \%$ dos casos para o ângulo anterior e $12 \%$ para o posterior).

Recentemente, técnicas de biologia molecular envolvendo a detecção de material fetal em amostras de sangue materno têm apresentado resultados superiores aos da ultra-sonografia no diagnóstico precoce do sexo fetal ${ }^{11-13}$. A pesquisa de DNA livre no plasma materno por meio da reação em cadeia de polimerase parece apresentar resultados mais confiáveis que a pesquisa de células fetais na circulação materna ${ }^{11-13}$. Além de apresentar elevada acurácia (98-99\%), esta pesquisa pode ser realizada a partir da oitava semana de gravide ${ }^{11}$

Apesar do elevado desempenho diagnóstico e de já se encontrar disponivel em nosso meio, trata-se de método de alto custo e disponivel em poucos centros. Além disso, o valor preditivo deste método diagnóstico é mais elevado quando o resultado do teste indica sexo masculino do que quando o teste indica sexo feminino ${ }^{14}$. Ou seja, há ainda um pequeno, mas significativo, número de er- 
ros diagnósticos quando o sexo fetal verdadeiro é o masculino mas o resultado do teste indica feminino. Assim sendo, especialmente em fetos com risco de doenças ligadas ao cromossomo $\mathrm{X}$, ainda é necessária a confirmação do sexo fetal, seja por meio de biópsia de vilo corial, seja por meio de ultra-sonografia após a $14^{\mathrm{a}}$ semana de gestação ${ }^{14}$. Outra alternativa disponivel para casais com antecedente de doença ligada ao X é o diagnóstico pré-implantacional. Estudos preliminares indicam bons resultados com essa técnica ${ }^{15}$. Não obstante, os custos envolvidos com a tecnologia de reprodução assistida tornam essa opção inacessivel a grande número de pessoas.

Concluímos que a acurácia do diagnóstico ultra-sonográfico do sexo fetal antes da $14^{\mathrm{a}}$ semana de gestação por meio da medida dos ângulos anterior e posterior do apêndice genital ainda é insuficiente para sua aplicação em fetos com risco de doenças graves ligadas ao $\mathrm{X}$, pois a certeza do sexo fetal ocorreu em uma minoria dos casos. Para estes fetos, a pesquisa de DNA fetal na circulação materna parece apresentar resultados mais precoces e mais confiáveis que os da ultrasonografia.

\section{Referências}

1. Benott B. Early fetal gender determination. Ultrasound Obstet Gynecol. 1999;13(5):299-300.

2. Lev-Toaff AS, Ozhan S, Pretorius D, Bega G, Kurtz AB, Kuhlman K. Three-dimensional multiplanar ultrasound for fetal gender assignment: value of the mid-sagittal plane. Ultrasound Obstet Gynecol. 2000;16(4):345-50.

3. Efrat $Z$, Akinfenwa OO, Nicolaides KH. First-trimester determination of fetal gender by ultrasound. Ultrasound Obstet Gynecol. 1999;13(5):305-7.

4. Mazza V, Contu G, Falcinelli C, Battafarano S, Cagnacci A, Vito G, et al. Biometrical threshold of biparietal diameter for certain fetal sex assignment by ultrasound. Ultrasound Obstet Gynecol. 1999;13(5):308-11.

5. Schupp TR, Brizot ML, Toyama J, Sato L, Watanabe L, Miyadahira S, et al. Identificação ultra-sonográfica do sexo fetal entre a $11^{\text {a }}$ e a $14^{a}$ semana de gestação. Rev Bras Ginecol Obstet. 2001;23(4):247-51.
6. Mazza V, Di Monte I, Pati M, Contu G, Ottolenghi C, Forabosco A, et al. Sonographic biometrical range of external genitalia differentiation in the first trimester of pregnancy: analysis of 2593 cases. Prenat Diagn. 2004;24(9):677-84.

7. Mazza V, Falcinelli C, Paganelli S, Contu G, Mantuano SM, Battafarano SD, et al. Sonographic early fetal gender assignment: a longitudinal study in pregnancies after in vitro fertilization. Ultrasound Obstet Gynecol. 2001;17(6):513-6.

8. Whitlow BJ, Lazanakis MS, Economides DL. The sonographic identification of fetal gender from 11 to 14 weeks of gestation. Ultrasound Obstet Gynecol. 1999;13(5):301-4.

9. Monteagudo A, Timor-Tritsch IE. First trimester anatomy scan: pushing the limits. What can we see now? Curr Opin Obstet Gynecol. 2003;15(2):131-41.

10.Bland JM, Altman DG. Statistical methods for assessing agreement between two methods of clinical measurement. Lancet. 1986;1(8476): 30710.

11.Levi JE, Wendel S, Takaoka DT. Determinação prénatal do sexo fetal por meio da análise de DNA no plasma materno. Rev Bras Ginecol Obstet. 2003;25(9):687-90.

12. Bianchi DW, Simpson JL, Jackson LG, Elias S, Holzgreve W, Evans MI, et al. Fetal gender and aneuploidy detection using fetal cells in maternal blood: analysis of NIFTY I data. National Institute of Child Health and Development Fetal Cell Isolation Study. Prenat Diagn. 2002;22(7):609-15.

13. Costa JM, Benachi A, Gautier E, Jouannic JM, Ernault P, Dumez Y. First-trimester fetal sex determination in maternal serum using real-time PCR. Prenat Diagn. 2001;21(12):1070-4.

14. Mazza V, Falcinelli C, Percesepe A, Paganelli S, Volpe A, Forabosco A. Non-invasive first trimester fetal gender assignment in pregnancies at risk for $\mathrm{X}$-linked recessive diseases. Prenat Diagn. 2002;22(10):919-24.

15. Tachdjian G, Frydman N, Audibert F, Ray P, Kerbrat V, Ernault P, et al. Clinical applications of fetal sex determination in maternal blood in a preimplantation genetic diagnosis centre. Hum Reprod. 2002;17(8):2183-6. 